\title{
Oleoylation of Wheat Straw Hemicelluloses in New Homogeneous System
}

\author{
RunCang Sun, ${ }^{\dagger}$ Jeremy Tomkinson, JianChao Liu, ${ }^{*}$ \\ and ZengChao GENG* \\ The BioComposites Centre, University of Wales, Bangor, Gwynedd, LL57 2UW, U.K. \\ * The North-western University of Agricultural \& Forest Sciences, \\ Yangling, People's Republic of China
}

(Received March 1, 1999)

\begin{abstract}
Oleoylated hemicelluloses with degrees of substitution between 0.24 and 1.67 were prepared by a rapid esterification of wheat straw hemicelluloses with oleoyl chloride using 4-dimethylaminopyridine as a catalyst and triethylamine as a neutralizer in homogeneous $N, N$-dimethylformamide and lithium chloride system. The effects of oleoyl chloride concentration, reaction duration, and triethylamine concentration have been studied. Under an optimum condition (sample 12 , molar ratio $1: 3$ ), over $90 \%$ of the free hydroxyl groups in native hemicelluloses were oleoylated in $35 \mathrm{~min}$ at $75^{\circ} \mathrm{C}$. The new materials were characterized by FT-IR, GPC, and thermal analysis. The molecular weight measurements (39700-110900) showed only a minimal degradation of hemicellulosic chains during the rapid reactions at $75^{\circ} \mathrm{C}$ for $15-40 \mathrm{~min}$. KEY WORDS Homogeneous System / Wheat Straw / Hemicelluloses / Oleoylation /
\end{abstract}

In recent years, studies concerning the total or partial substitution of synthetic plastics in key applications by biodegradable materials have been increasing. ${ }^{1}$ Particularly, replacement of petroleum based plastics with materials from agricultural residues such as straw and grass is attractive from the standpoint of making the end product biodegradable. Wheat straw is the most abundant residues among the crops. About 170 million tons of wheat straw are produced yearly in Europe. ${ }^{2}$ Part of the straw has been used for making paper, particle board, furniture and building panels, and other pressed forms as substitutes or supplements to wood-based materials or plastics made from petrochemicals, especially in countries where wood supplies are limited such as in China, India, and Malaysia, but a major part of the straw is discarded as a waste product. ${ }^{3}$

The predominating constituents of wheat straw are cellulose $(35-40 \%)$ and hemicelluloses $(30-35 \%) .{ }^{4}$ The latter consists of L-arabino-D-xylans with various branching degrees. The main polysaccharide chain consists of D-xylopyranose units linked by a glycosidic $\beta(1 \rightarrow 4)$ bond. The side chains are formed by L-arabinofuranose units linked by an $\alpha(1 \rightarrow 3)$ bond. The uronic acid, mainly 4-O-methyl-D-glucuronic acid (MeGlcA), is bound to D-xylose units of the main chain by an $\alpha(1 \rightarrow 2)$ bond. The hemicelluloses were, in general, contaminated in a small extent by neutral polysaccharides containing glucose and galactose units. Some trace amounts of rhamnose and mannose were also found in the neutral fractions. ${ }^{5}$ However, due to the hydrophilic nature of hemicelluloses, blends with hydrophobic plastics have poor mechanical properties due to the poor interfacial adhesion. The hydrophilicity of hemicelluloses results in their inability to form a continuous phase with the synthetic polymer. These shortcomings can be overcome by their modification such as partial hydrolysis, oxidation, reduction, etherification or esterification of the hydroxyl groups, and cross-linking.

One way to approach miscibility and improving ad- hesion of phase is to replace the hydrophilic -OH groups of hemicelluloses with hydrophobic groups through esterification. The acetate prepared from white birch xylans displayed molecular association in chloroform. ${ }^{6}$ The nitrates of undegraded xylans were insoluble in common solvents and decomposed readily at room temperature. ${ }^{7}$ Philipp et al. ${ }^{8}$ studied the sulfation of xylans dissolved in $\mathrm{N}_{2} \mathrm{O}_{4}-\mathrm{N}, \mathrm{N}$-dimethylformamide (DMF) solution and observed little difference between the $2-\mathrm{OH}$ and $3-\mathrm{OH}$ groups in reactivity. Other studied the crosslinking, cyanoethylation, and oxidation of hemicelluloses to give products with different ion exchange capacities or as a binder for paper making. ${ }^{9}$

However, due to the hydrophilic nature in hemicelluloses, a number of hemicellulose derivatives is currently prepared under heterogeneous conditions. Aside from the inherently unfavorable reaction kinetics, some problems such as poor uniformity of substitution, low yields and extensive by-product formation arise during the heterogeneous solutions. In addition, the isolation and characterization of the product derivatives are also tedious. ${ }^{10,11}$ Organic esters of xylans were generally prepared by first dispersing or dissolving them in anhydrous formamide followed by the addition of acylating agents without swelling. ${ }^{12}$ This process, in general, gave the low yield of the products with a long time reaction period.

In the present study, we report optimized synthetic conditions for preparing oleoylated hemicelluloses in homogeneous solution of $\mathrm{DMF} /$ lithium chloride $(\mathrm{LiCl})$. The modified hemicelluloses are expected to exhibit an increased hydrophobic character due to the incorporation of long oleoyl groups. Furthermore, the oleoylated hemicelluloses are expected to show different thermal behavior due to the replacement of hydroxyl groups with ester groups. The products are characterized by yield of oleoylation, degree of substitution (DS), FT-IR spectroscopy, molecular size, solubility, and thermal analysis.

\footnotetext{
† To whom correspondence should be addressed.
} 


\section{MATERIALS AND METHODS}

\section{Materials}

Wheat straw was provided by Compak Co. (Gainsborough, England). The straw contained $14.2 \%$ lignin, $38 \%$ cellulose, and $32 \%$ hemicelluloses on a dry basis. The amount of proteins, pectins, and waxes in this straw was insignificant. The straw was first cut into $1-2 \mathrm{~cm}$ length by hand, and then ground to pass through a $0.7 \mathrm{~mm}$ screen and stored at $5^{\circ} \mathrm{C}$ until use. Oleoyl chloride, 4-dimethylaminopyridine (DMAP), and triethylamine (TEA) were reagent grade. DMF solvent was dried prior to use according to conventional methods. Anhydrous $\mathrm{LiCl}$ was obtained by dry at $130^{\circ} \mathrm{C}$ for $2 \mathrm{~h}$ before use.

\section{Isolation and Characterization of Hemicelluloses}

The scheme for extraction and isolation of hemicelluloses from wheat straw is shown in Figure 1. The ground straw was first delignified with sodium chlorite in acetic solution ( $\mathrm{pH} 4.2$, adjusted by $10 \%$ acetic acid) at $75^{\circ} \mathrm{C}$ for $2 \mathrm{~h}$. The hemicelluloses were then obtained from the delignified sample by extraction with $10 \%$ potassium hydroxide for $19 \mathrm{~h}$ at $25^{\circ} \mathrm{C}$ with a liquor ratio of $1: 20$. The solubilized hemicelluloses were isolated by precipitation of the neutralized hydrolysate in 3 volumes of $95 \%$ ethanol. After filtration, the pellets of the hemicelluloses were washed by $70 \%$ ethanol and air-dried.

The neutral sugar composition of the isolated hemicelluloses was determined by gas chromatography (GC) analysis of their alditol acetates. ${ }^{13}$ Alkaline nitrobenzene oxidation of residual lignin from hemicelluloses was performed at $170^{\circ} \mathrm{C}$ for $3 \mathrm{~h}$. The lignin content in hemicelluloses was calculated by 2.41 multiplying the yield of phenolics, obtained by nitrobenzene oxidation. ${ }^{14}$ Methods of uronic acid analysis, determination of phenolic acids and aldehydes in nitrobenzene oxidation mixtures with high performance liquid chromatography (HPLC), and measurement of the native hemicellulosic molecular weight have been described in previous papers. ${ }^{4,15,16}$

FT-IR spectra were obtained on an FT-IR (Nicolet 750) spectrophotometer using a $\mathrm{KBr}$ disc containing $1 \%$ finely ground samples. The solution-state ${ }^{13} \mathrm{C}$ NMR spectrum was obtained on a Bruker $250 \mathrm{AC}$ spectrometer operating in the FT mode at $62.4 \mathrm{MHz}$ under total proton decoupled conditions. It is recorded at $25^{\circ} \mathrm{C}$ from $200 \mathrm{mg}$ of sample dissolved in $1.0 \mathrm{~mL} \mathrm{D}_{2} \mathrm{O}$ after 12000 scans. A $60^{\circ}$ pulse flipping angle, a $3.9 \mu$ s pulse width and $0.85 \mathrm{~s}$ acquisition time were used.

\section{Oleoylation of Hemicelluloses}

After being swollen in water, the hemicelluloses were esterified in homogeneous $\mathrm{DMF} / \mathrm{LiCl}$ solvent system. The general procedure of esterification was as follows. The hemicelluloses $(0.6 \mathrm{~g}, 0.008 \mathrm{~mol}$ of hydroxyl functionality) were placed in a two necked flask equipped with an automatic stirrer and a condenser. Thirty $\mathrm{mL}$ distilled water was added and the solution was heated to $80^{\circ} \mathrm{C}$ until completely dissolved (approximately $5 \mathrm{~min}$ ). After addition of $30 \mathrm{~mL}$ DMF, the mixture was stirred for another $5 \mathrm{~min}$ at $80^{\circ} \mathrm{C}$. The water was then removed from the swollen gel by repeated distillation under reduced pressure at $50^{\circ} \mathrm{C}$. $\mathrm{LiCl}(0.15 \mathrm{~g})$ and DMAP $(0.10 \mathrm{~g})$ were added and the reactor was kept at $75^{\circ} \mathrm{C}$ under stirring. Then $1.32 \mathrm{~mL}(0.004 \mathrm{~mol}$ of carboxyl functionality, molar ratio $1: 1), 1.98 \mathrm{~mL}(0.006 \mathrm{~mol}$ of carboxyl functionality, molar ratio $1: 1.5), 2.64 \mathrm{~mL}$ $(0.008 \mathrm{~mol}$ of carboxyl functionality, molar ratio $1: 2)$, $3.30 \mathrm{~mL}(0.010 \mathrm{~mol}$ of carboxyl functionality, molar ratio $1: 2.5)$, and $3.96 \mathrm{~mL}(0.012 \mathrm{~mol}$ of carboxyl functionality,

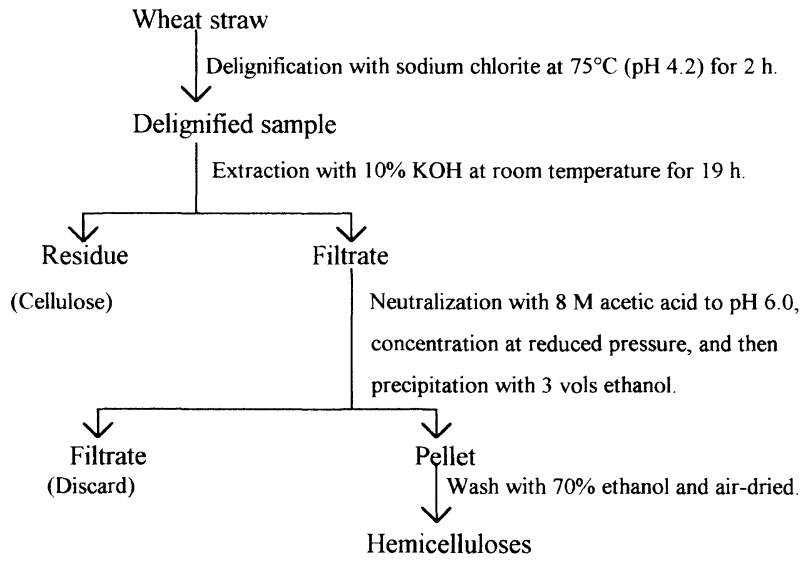

Figure 1. Scheme for extraction of hemicelluloses from wheat straw.

Table I. The yield ${ }^{\mathrm{a}}$ of oleoylated hemicelluloses and the degree of substitution (DS)

\begin{tabular}{|c|c|c|c|c|c|c|}
\hline \multicolumn{4}{|c|}{ Oleoylation conditions } & \multicolumn{3}{|c|}{ Oleoylated hemicelluloses } \\
\hline Molar ratio $^{b}$ & Temperature & Time $/ \mathrm{min}$ & TEA $/ \%^{c}$ & Sample & Yield $/ \%$ & DS \\
\hline $1: 1$ & $75^{\circ} \mathrm{C}$ & 15 & 56 & 1 & 29.6 & 0.24 \\
\hline $1: 1$ & $75^{\circ} \mathrm{C}$ & 20 & 56 & 2 & 31.6 & 0.29 \\
\hline $1: 1.5$ & $75^{\circ} \mathrm{C}$ & 15 & 83 & 3 & 37.6 & 0.44 \\
\hline $1: 1.5$ & $75^{\circ} \mathrm{C}$ & 30 & 83 & 4 & 39.8 & 0.50 \\
\hline $1: 2.0$ & $75^{\circ} \mathrm{C}$ & 20 & 90 & 5 & 41.3 & 0.53 \\
\hline $1: 2.0$ & $75^{\circ} \mathrm{C}$ & 25 & 110 & 6 & 42.2 & 0.56 \\
\hline $1: 2.0$ & $75^{\circ} \mathrm{C}$ & 20 & 110 & 7 & 42.9 & 0.57 \\
\hline $1: 2.0$ & $75^{\circ} \mathrm{C}$ & 30 & 110 & 8 & 44.6 & 0.62 \\
\hline $1: 2.0$ & $75^{\circ} \mathrm{C}$ & 35 & 110 & 9 & 55.2 & 0.88 \\
\hline $1: 2.0$ & $75^{\circ} \mathrm{C}$ & 40 & 110 & 10 & 56.5 & 0.91 \\
\hline $1: 2.5$ & $75^{\circ} \mathrm{C}$ & 30 & 140 & 11 & 71.9 & 1.30 \\
\hline $1: 3$ & $75^{\circ} \mathrm{C}$ & 35 & 174 & 12 & 86.8 & 1.67 \\
\hline
\end{tabular}

a Based on assumption that all of the hemicelluloses are converted to di-oleoylated hemicelluloses (yield, $100 \% ; \mathrm{DS}, 2.0$ ). If no reaction occurred and all of the hemicelluloses were recovered unreacted, the yield percentage would be $20.0 \%$ (DS, 0.0 ). ${ }^{\mathrm{b}}$ Molar ratio represents the mol of xylose in hemicelluloses $/ \mathrm{mol}$ of oleoyl chloride. ${ }^{\mathrm{c}}$ TEA (\%) represents the weight percentage of hemicelluloses (w/w). 


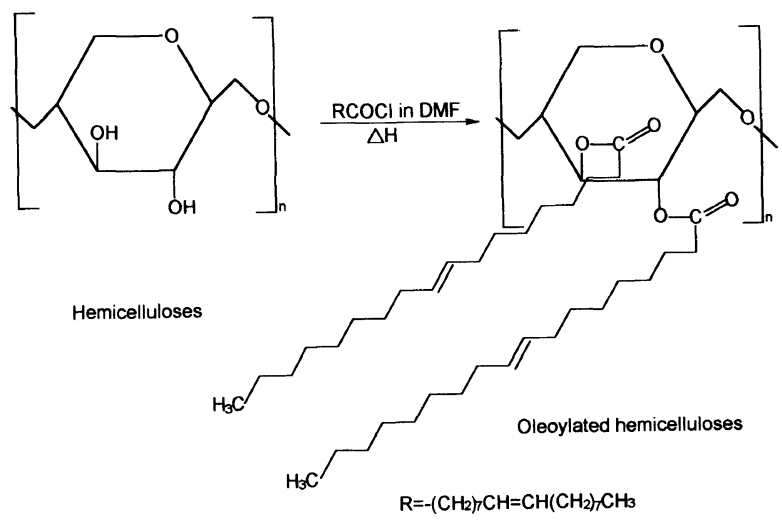

Scheme 1. Oleoylation of wheat straw hemicelluloses.

molar ratio $1: 3$ ) oleoyl chloride with a required amount of TEA ( $\%$ of the native hemicelluloses, w/w, Table I) in $10 \mathrm{~mL}$ DMF were, respectively, added dropwise, and the homogeneous reaction mixture was stirred for a total period of $10,15,20,25,30,35$, and $40 \mathrm{~min}$ at $75^{\circ} \mathrm{C}$, respectively. Upon completion of the reaction, the mixture was cooled and poured with vigorous stirring into a glass beaker containing $120 \mathrm{~mL} 95 \%$ ethanol to precipitate the esterified hemicelluloses and to eliminate any color impurities. The product was washed twice with $100 \mathrm{~mL}$ ethanol and acetone, respectively. The excess of ethanol and acetone was removed by an air stream and the hemicellulose ester was dried at $55^{\circ} \mathrm{C}$ overnight and its weight was measured.

\section{Characterization of the Oleoylated Hemicelluloses}

The yield percentages were calculated based on the assumption that all of the hemicelluloses were converted to di-oleoylated hemicelluloses (Scheme 1). In the case the yield percentage would be $100 \%$. The degree of substitution (DS) for a hemicellulose ester is defined as the moles of substituents of hydroxyl groups per D-xylopyranosyl structural unit of the hemicellulosic polymer; with two hydroxyl groups per unit. The theoretical maximum DS is 2.0. The unreacted oleoyl chloride in the mixture of reactions was separated from the product by dissolving in $95 \%$ ethanol and acetone. If no reaction occurred and all of the hemicelluloses were recovered unreacted, the yield percentage and the degree of substitution would be $20.0 \%$ and 0.0 , respectively. The solubility was measured at $3.0 \%$ concentration in different organic solvents.

The weight-average molecular weights of oleoylated hemicelluloses were determined by gel permeation chromatography on a PLgel $5 \mu$ Mixed-D column. The samples were dissolved in pyridine/ $\mathrm{LiCl}(0.1 \%, \mathrm{w} / \mathrm{w})$ at a concentration of $0.1 \%$, and a $200 \mu \mathrm{L}$ sample in solution was injected. The column was operated at $80^{\circ} \mathrm{C}$ and eluted with pyridine $/ \mathrm{LiCl}$ at a flow rate of $1 \mathrm{~mL} \mathrm{~min}^{-1}$. Detection was achieved with a Knauer differential refractometer. The column was calibrated using PL pullulan polysaccharide standards.

\section{RESULTS AND DISCUSSION}

\section{Characterization of the Isolated Hemicelluloses}

Treatment of the delignified wheat straw with $10 \%$
$\mathrm{KOH}$ at ambient temperature for $19 \mathrm{~h}$ yielded $31.7 \%$ hemicelluloses. The neutral sugar composition showed that xylose is a predominant component sugar, which comprised $82.9 \%$ of the total sugars. Arabinose $(9.7 \%)$, appeared as the second major sugar component. Glucose $(4.0 \%)$, galactose $(2.2 \%)$, and rhamnose $(1.1 \%)$ were present as minor sugar constituents. The uronic acids, mainly 4-O-methyl-D-glucuronic acid (MeGlcA), were present in a noticeable amount $(4.6 \%)$. Gel permeation chromatography (GPC) showed the native hemicelluloses had a weight-average molecular weight of 27900 with a polydispersity of 5.2. The presence of associated lignin in the isolated hemicelluloses was confirmed by alkaline nitrobenzene oxidation and HPLC analysis. Obviously, the major phenolic monomer component in the oxidation mixture was found to be vanillin $(0.17 \%, w / w)$, which represented $36 \%$ of the total phenolic acids and aldehydes. This suggested that the lignins in the wheat straw cell walls are linked to hemicelluloses mainly with guaiacyl units. Other small amounts of syringic acid $(0.059 \%)$, p-coumaric acid $(0.054 \%)$, vanillic acid $(0.048 \%)$, syringaldehyde $(0.053 \%), p$-hydroxybenzaldehyde $(0.050 \%)$, ferulic acid $(0.026 \%)$, and $p$-hydroxybenzoic acid $(0.010 \%)$ were also identified in the mixture produced by nitrobenzene oxidation.

In order to characterize the structural features of the native hemicelluloses, the isolated hemicelluloses were analyzed by ${ }^{13} \mathrm{C}$ NMR spectroscopy (Figure 2). This allows elucidation of the polymer backbone and can be employed to evaluate the type of side chains branching along the backbone. ${ }^{17,18}$ The spectrum was interpreted on the basis of reported data for structurally-defined arabinoxylan-type, glucoronoxylan-type and L-arabino(4-O-methyl-D-glucurono)-D-xylan, as well as those of wheat straw hemicelluloses extracted before delignification. ${ }^{16,17,19-21}$ The main 1,4-linked $\beta$-D-xylopyranosyl (Xylp) units are obviously characterized by the signals at $105.1,78.5,77.6,76.0$, and $65.9 \mathrm{ppm}$, which attributes to $\mathrm{C}-1, \mathrm{C}-4, \mathrm{C}-3, \mathrm{C}-2$, and $\mathrm{C}-5$ of the $\beta$-D-Xylp units, respectively. The signals at $112.0,89.1,83.0,81.0$, and $64.4 \mathrm{ppm}$ correspond to C-1, C-4, C-2, C-3, and C-5 of $\alpha$-L-arabinofuranosyl (Araf) residues, respectively. C-1 in $1,3,4$ or 1,2,4-linked $\beta$-D-Xylp units appeared at $103.4 \mathrm{ppm}$ (data not shown). Three signals at 175.6, 85.0, and $58.8 \mathrm{ppm}$ (data not shown) originate from C-6, C-4 and 4-O-methoxyl group of glucuronic acid residue in the xylan. The signal at $26.1 \mathrm{ppm}$ related to $-\mathrm{CH}_{3}$ in MeGlcA or in $\mathrm{Ar}-\mathrm{COCH}_{3}$, resulting from the associated lignins. The signal at $184.1 \mathrm{ppm}$ originates the carboxylic group in salts of MeGlcA or in $\alpha, \beta$-unsaturated acid or ester $\left(\mathrm{Ar}-\mathrm{HC}=\mathrm{CH}-\mathrm{COO}^{-}\right)$such as esterified ferulic or $p$-coumaric acids in native hemicelluloses. These signals with further methylation analysis clearly showed that $\alpha-(1 \rightarrow 2)$-linked MeGlcA unit and $\alpha-(1 \rightarrow 3)$-linked L-Araf and D-Xylp units represented the main side chains of the xylan backbone. ${ }^{16}$

\section{Yield and Degree of Substitution}

The yield percentage and DS are given in Table I. Due to the lack of associated unreacted oleoyl chloride in isolated products, the DS values of the modified hemicelluloses were determined from the yield percentage of oleoylated hemicelluloses based on a theoretical value 


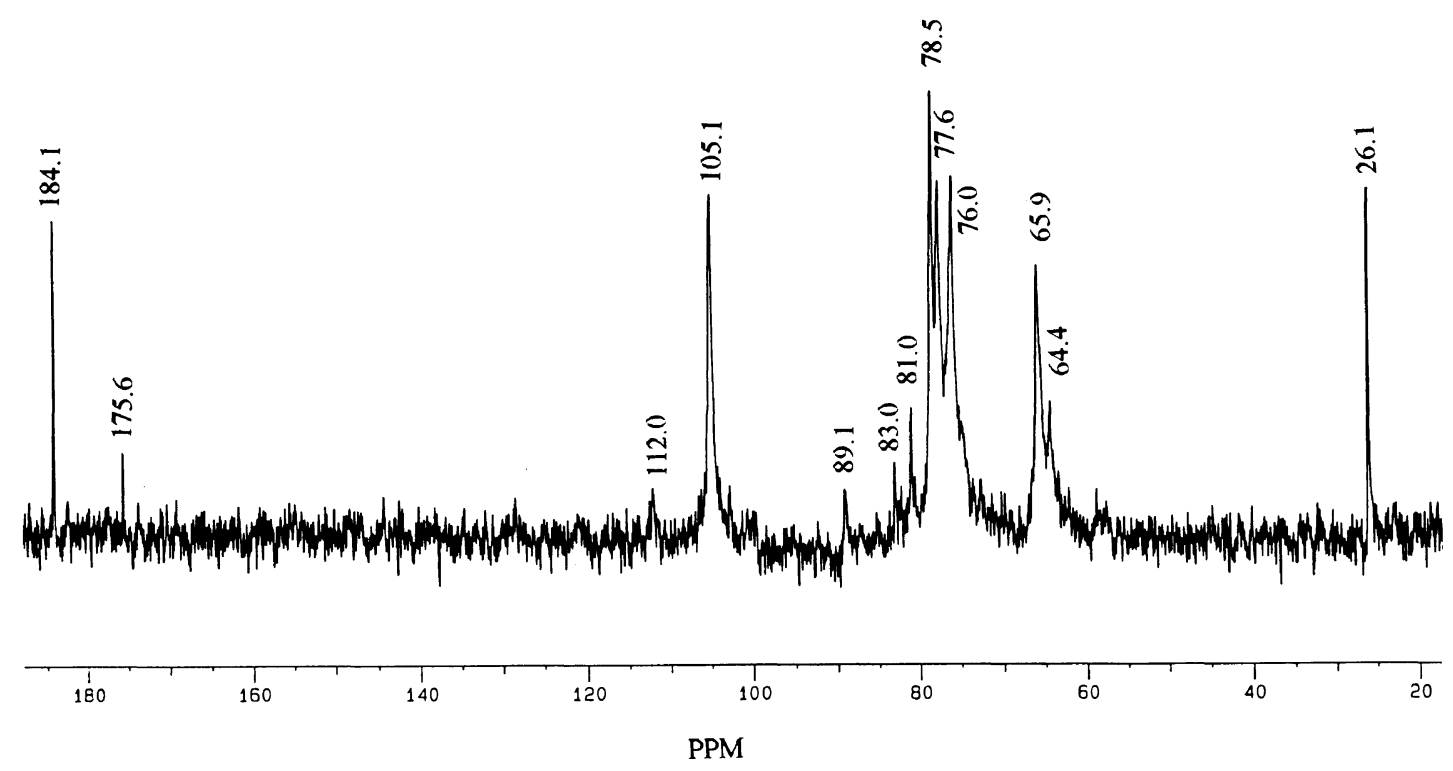

Figure 2. ${ }^{13} \mathrm{C}$ NMR spectrum (in $\mathrm{D}_{2} \mathrm{O}$ ) of native hemicelluloses extracted with $10 \% \mathrm{KOH}$ at $25^{\circ} \mathrm{C}$ for $19 \mathrm{~h}$ from delignified wheat straw.

of two oleoyl per repeat xylose unit. As can be seen in Table I, the yield percentages varied from 29.6 to $86.8 \%$ with DS values between 0.24 and 1.67. Obviously, the yield and DS of the products depended on the molar ratio of reactant agents, reaction duration, and amounts of TEA used. Increases of molar ratio from 1:1 (sample 1) to $1: 3$ (sample 12 ), reaction duration from 15 to $35 \mathrm{~min}$, and TEA concentration from $56 \%$ to $174 \%$ resulted in a significantly increasing yield from less $30 \%$ to over $80 \%$ and a DS value from below 0.3 to over 1.6, respectively. This increase could be interpreted in terms of greater availability of oleoyl chloride molecules in the proximity of the hemicellulosic molecules at higher concentration of the esterifying agent. ${ }^{22}$ It is probable that the hemicellulosic hydroxyl are immobile and their reaction will therefore rely on the availability of the oleoyl chloride molecules in the vicinity of hydroxyl groups. Similar trend was observed for acetylation of maize starch. ${ }^{22}$ Table I also shows that the yield and DS depend on the reaction duration. Clearly, the yield of oleoylation and DS value increased with the prolonging reaction period. A prolonging reaction period from 20 to $40 \mathrm{~min}$ in samples $7-10$ led to increment of yield from $42.9 \%$ to $56.5 \%$ and DS from 0.57 to 0.91 , respectively. This enhancement of oleoylation by prolonging duration of the reaction was a direct consequence of the favorable effect of time on diffusion and adsorption of the reactants between the esterifying agent and the hemicellulose molecules. It is, therefore, an optimum oleoylation of wheat straw hemicelluloses in the reaction conditions given would be as follows: molar ratio $1: 3$, temperature $75^{\circ} \mathrm{C}$, reaction duration $35 \mathrm{~min}$, and TEA concentration $174 \%$.

The esterification of hemicelluloses in a heterogeneous process relied on the advancement of the reaction from layer to layer through the hemicellulose structure, which resulted in a low yield product with a non-uniform substitution during the derivatizations (unpublished data). In order to improve these shortages, we studied oleoylation reaction of hemicelluloses in homogeneous $\mathrm{DMF} / \mathrm{LiCl}$ system, using acyl to exchange hemicellulose
$\mathrm{O}-\mathrm{H}$ protons. It was found that the first dissolution of native hemicelluloses in water and then treatment in a dipolar-aprotic solvent such as $\mathrm{DMF} / \mathrm{LiCl}$ system can lead to a highly swollen gel suspension of the polymer and activate the polymers. Owing to their property of forming hydrogen bonds, strongly polar aprotic solvents are able to prevent the aggregation of flexible hemicellulose chains, promoting the interactions between substrate and reagents. ${ }^{17}$ Another obvious advantage of this solvent system for the preparation of hemicellulose derivatives lied in the ability to conduct a variety of organic reactions, producing high degree of substitution under mild conditions, and increasing the efficiency of such reactions in homogeneous solution. Additionally, $\mathrm{DMF} / \mathrm{LiCl}$ acted as the solvent for the oleoylated hemicelluloses, which would ensure not only high substitution but also more uniform substitution due to greater accessibility of the reagent. ${ }^{10}$ Furthermore, the lower reagent quantities required and a rapid reaction process were other two significant advantages for the esterification of hemicelluloses in homogeneous $\mathrm{DMF} / \mathrm{LiCl}$ system.

Our previous studies on native hemicelluloses showed that hemicelluloses, extracted with $0.5 \mathrm{M} \mathrm{NaOH}$ at $30^{\circ} \mathrm{C}$ for $2 \mathrm{~h}$ from lignified wheat straw, was to be essentially a $(1 \rightarrow 4)$ linked $\beta$-D-xylan with $4-O$-methyl-D-glucopyranosyluronic acid attached at position 2 , and $\mathrm{L}$-arabinofuranosyl and D-xylopyranosyl groups attached at position 3. For every $13 \mathrm{D}$-xylopyranosyl residues in the main chain, there is one L-arabinofuranosyl group, for every 18 such D-xylopyranosyl residues, there is one D-xylopyranosyl group, and for approximately 26 such D-xylopyranosyl residues, there is one MeGlcA unit. ${ }^{16}$ Based on this branched structure of hemicelluloses, for every $20 \mathrm{D}$-xylopyranosyl residues in the backbone, there are 3 side chains attached at C-2 or C-3 of xylose in the main chain. This resulted in only 37 free hydroxyl groups that can be oleoylated, which accounted for a maximum yield value less than $100 \%, 94.0 \%$ and a maximum DS value less than $2.0,1.85$. In sample 12 , a maximum yield of $86.8 \%$ and a highest DS value of 1.67 indicated 
<smiles>[R]C(=O)[n+]1ccc(N(C)C)cc1</smiles><smiles>[R]C(=O)[n+]1ccc(N(C)C)cc1</smiles><smiles>[R][R](=O)[CH-][OH+]C([R])=O</smiles>

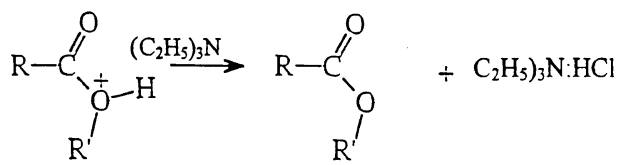

Scheme 2. Mechanism of oleoylation of wheat straw hemicelluloses.

that over $90 \%$ of the free hydroxyl groups in native hemicelluloses were oleoylated under the reaction condition used. Further work is in progress to gain a complete substitution (DS, 1.85) by addition of more mol of oleoyl chloride permol of free $\mathrm{OH}$ groups or by prolonging the reaction duration.

Studies on reaction mechanism showed that the oleoylation process involved an attacking of acyl carbon centre by nucleophile such as DMAP and a removal of hydrochloric acid (Scheme 2). It was found that DMAP, a widely used versatile hypernucleophilic acylation catalyst, was $10^{4}$ times more active than pyridine during the esterification process. Similarly TEA, used as a neutralizer, had a more significant efficiency than the pyridine. This is largely due to the much stronger basic property of TEA than that of pyridine. Addition of TEA can counteract the hydrochloric acid, produced during the esterification process, by forming amine salt and stabilize the reaction system $\mathrm{pH}$ between 5.5 and 6.5 . These effects significantly lessen the chance of degradation of the hemicellulose backbone by acid hydrolysis and therefore enhance the oleoylation. The present work is the first part of a more extended study undertaken by our laboratories towards investigating a rapid method for esterification of wheat straw hemicelluloses in homogeneous system.

\section{FT-IR Spectra}

The FT-IR spectra of native (spectrum a) and oleoylated (spectrum b, sample 11) hemicelluloses are shown in Figure 3. In the native hemicellulosic spectrum the characteristic broad peak of hemicelluloses appears at $1047 \mathrm{~cm}^{-1}$. This peak is attributed to $\mathrm{C}-\mathrm{OH}$ bond stretching. A sharp band at $898 \mathrm{~cm}^{-1}$ is characteristic of $\beta$-glucosidic linkages between the sugars units. ${ }^{23}$ This confirmed that the xylose residues forming the backbone of the macromolecule are linked by $\beta$ form bonds. A

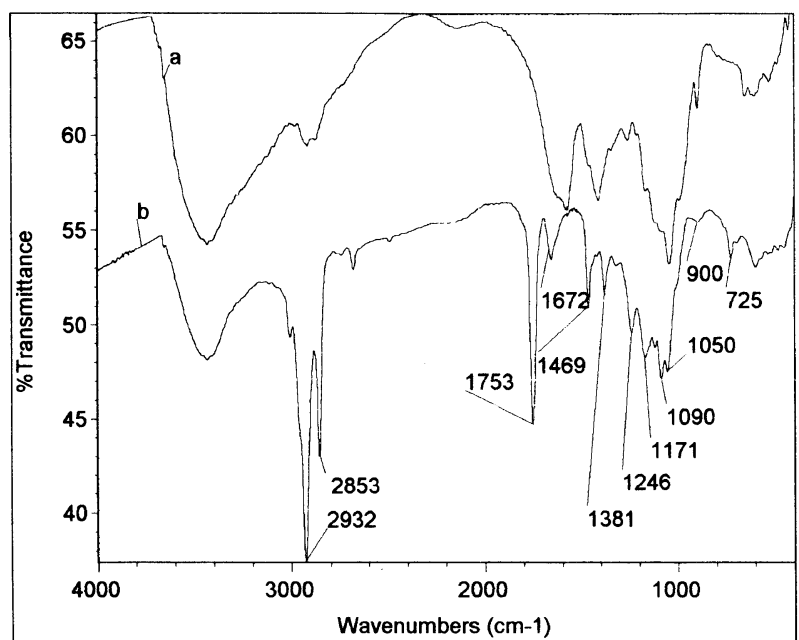

Figure 3. FT-IR spectra of native hemicelluloses (spectrum a) and oleoylated hemicellulosic sample 11 (spectrum b).

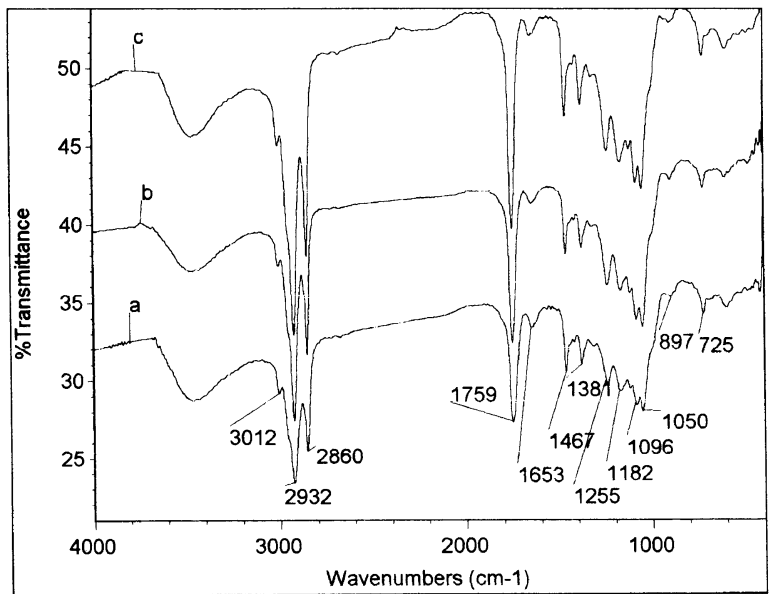

Figure 4. FT-IR spectra of oleoylated hemicellulosic samples 3 (spectrum a), 6 (spectrum b), and 9 (spectrum c).

strong broad band due to hydrogen bonded hydroxyls appears at $3400 \mathrm{~cm}^{-1}$ and the symmetric $\mathrm{C}-\mathrm{H}$ vibration band at 2852 and $2930 \mathrm{~cm}^{-1}$. Other prominent bands corresponding to native hemicelluloses appear at 1670 , $1580,1470,1418,1260,1176,1094$, and $985 \mathrm{~cm}^{-1}$. The oleoylation reactions were monitored by observing in the infrared spectra a reduction in the oleoyl chloride carbonyl absorbance at $1800 \mathrm{~cm}^{-1}$ and the appearance of an ester carbonyl absorbance at $1753 \mathrm{~cm}^{-1}$. The FT-IR spectrum of oleoylated hemicelluloses shows strong bands at $1753 \mathrm{~cm}^{-1}, 2853 \mathrm{~cm}^{-1}$, and $2932 \mathrm{~cm}^{-1}$, which are attributed to stretching deformation of the ester carbonyl group and the methyl/methylene groups, respectively. The strong band at $3400 \mathrm{~cm}^{-1}$ (hydroxyl groups) of native hemicelluloses decreases after the esterification reaction due to ester bond formation. The disappearance of peaks at $1800 \mathrm{~cm}^{-1}$ in spectrum $b$ indicated that the products are free of the unreacted oleoyl chloride. Another evidence of oleoylation by showing the presence of ester band appears at $1246 \mathrm{~cm}^{-1}$, which corresponds to $-\mathrm{C}-\mathrm{O}$ - stretching in ester bonds. ${ }^{24}$ The appearances of other three prominent bands at 1469 , 1381, and $1171 \mathrm{~cm}^{-1}$ in oleoylated hemicellulosic spectrum (b) are attributed to the $\mathrm{CH}_{2}$ bending, $\mathrm{C}-\mathrm{H}$ 
Table II. Weight-average $\left(\bar{M}_{w}\right)$ and number-average $\left(\bar{M}_{n}\right)$ molecular weights and polydispersity $\left(\bar{M}_{w} / \bar{M}_{n}\right)$ of the oleoylated hemicellulosic samples

\begin{tabular}{|c|c|c|c|c|c|c|c|c|c|c|c|c|}
\hline & \multicolumn{12}{|c|}{ Oleoylated hemicellulosic samples ${ }^{\mathrm{a}}$} \\
\hline & 1 & 2 & 3 & 4 & 5 & 6 & 7 & 8 & 9 & 10 & 11 & 12 \\
\hline $\bar{M}_{w}$ & 39700 & 40700 & 48300 & 50800 & 52900 & 54400 & 55900 & 59100 & 73900 & 75000 & 91400 & 110900 \\
\hline $\bar{M}_{n}^{n}$ & 37800 & 37700 & 46400 & 44600 & 49000 & 51300 & 53200 & 51400 & 62600 & 62500 & 79500 & 97300 \\
\hline $\bar{M}_{w} / \bar{M}_{n}$ & 1.05 & 1.08 & 1.04 & 1.14 & 1.08 & 1.06 & 1.05 & 1.15 & 1.18 & 1.20 & 1.15 & 1.14 \\
\hline
\end{tabular}

${ }^{\text {a }}$ Corresponding to the samples in Table I.

bending, and $\mathrm{C}-\mathrm{C}$ stretching, respectively. The absorption at $1670-1672 \mathrm{~cm}^{-1}$ in both spectra is principally associated with the $\mathrm{C}=\mathrm{O}$ stretch of carboxylic anion (salt) for MeGlcA in native hemicelluloses and products.

Figure 4 depicts the FT-IR spectra of oleoylated samples 3 (spectrum a), 6 (spectrum b), and 9 (spectrum c). There are no significant differences observed in the spectra (e.g., peak shifts), indicating the similar structure of the modified hemicelluloses. However, on closer examination the spectra, some small differences were clearly distinguished from the spectra. Obviously, the absorbance intensity of the signals for $\mathrm{C}=\mathrm{O}$ of the ester moieties of three samples depended on the DS. With an increment of DS value from 0.44 in sample 3 (spectrum a) to 0.56 in sample 6 (spectrum b), and to 0.88 in sample 9 (spectrum c), the absorption intensity for ester bond at $1759 \mathrm{~cm}^{-1}$ increased from sample 3 to sample 6 , and to sample 9 , corresponding to the growth of DS value. In addition, the absorbance bands at 2860/2932 for methyl/methylene groups enhanced from spectrum a to $\mathrm{b}$, and to $\mathrm{c}$ as the DS value raised from 0.44 to 0.56 , and to 0.88 in samples 3,6 , and 9 , respectively.

\section{Molecular Weight}

In order to illustrate whether the degradation occurred during the reaction in $\mathrm{DMF} / \mathrm{LiCl}$ system, all the molecular weights of oleoylated hemicelluloses were determined by GPC, and the weight-average $\left(\bar{M}_{w}\right)$ and number-average $\left(\bar{M}_{n}\right)$ molecular weights and polydispersity $\left(\bar{M}_{w} / \bar{M}_{n}\right)$ of the esterified hemicelluloses are given in Table II. As compared to the theoretical molecular weights, which were based on the molecular weight of the starting native hemicelluloses $\left(\bar{M}_{w}=27900\right)$ and the DS value, all of the molecular weights obtained were slightly lower than the expected values by $7 \%$, indicating that only a minimal degradation occurred during the reactions at $75^{\circ} \mathrm{C}$ with a short period of $15-40 \mathrm{~min}$. The elution profiles of oleoylated hemicellulose sample 4 is illustrated in Figure 5. The molecular weight distribution ranged between 95200 and 34200 with a main peak at 50800, indicating a narrow molecular range. This was consistent with the conclusions of only a little degradation occurred during the $\mathrm{DMF} / \mathrm{LiCl}$ homogeneous esterification condition given in this study.

\section{Solubility}

Esterification of oleoyl groups with the native hemicelluloses may open the structure of hemicelluloses. This together with the physical changes in the hemicellulosic structure and molecular degradation occurring

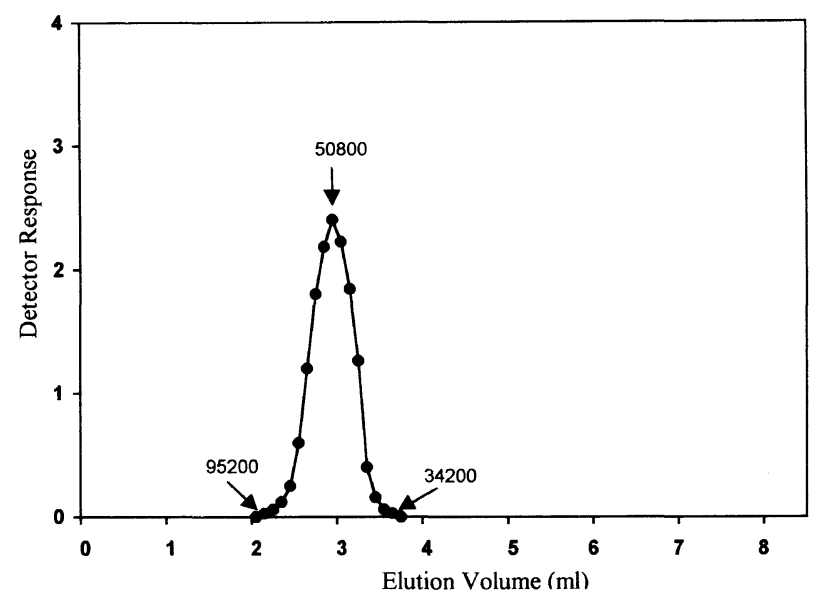

Figure 5. GPC molecular weight distribution of oleoylated hemicellulosic sample 4 .

during esterification would act in favor of solubility. That is, introduction of hydrophobic oleoyl groups in the molecular structure of hemicelluloses would be expected to alter their solubility properties. Such alteration would rely essentially on the degree of substitution. ${ }^{25,26}$ The solubility of the products was, therefore, investigated only using the oleoylated hemicellulose sample 11 with a DS value of 1.30 as a sample. The sample showed a good solubility in pyridine, and is partially soluble in dimethylsulfoxide (DMSO), chloroform, dichloromethane, toluene, and tetrahydrofuran (THF) at room temperature. This increasing hydrophobic capacity would lead to potential use of oleoylated hemicelluloses in the production of films and membranes from the solution by casting and evaporating of the solvent. This work is being further investigated.

In conclusion, oleoylation of the free hydroxyl groups from hemicelluloses in homogeneous $\mathrm{DMF} / \mathrm{LiCl}$ system using DMAP as a catalyst and TEA as a base represents a suitable, rapid, and effective method for the esterification of wheat straw hemicelluloses. The $\mathrm{DMF} / \mathrm{LiCl}$ acted as the solvent for both the native hemicelluloses and the products and ensured uniform substitution by great accessibility of the reagent. In addition, reactions to oleoylated hemicelluloses can be controlled with high accuracy by adjusting the TEA concentration and the molar ratio of reagent and hydroxyl functionality. Under an optimum reaction condition (sample 12, molar ratio $1: 3$, TEA $\% 174,75^{\circ} \mathrm{C}$, $35 \mathrm{~min}$ ), the product with a high DS value of $1.67 \mathrm{can}$ be obtained without significant degradation of the native hemicelluloses, in which over $90 \%$ of the free hydroxyl groups in native hemicelluloses were oleoylated. 
Acknowledgments. The authors are grateful for the financial support of this research from the China Bridge International (U.S.A.), National Natural Science Foundation of China, and European Community under the Industrial \& Materials Technologies Programme (BriteEuRam III)-Depolymerisation, Polymerisation, and Applications of Biosustainable Raw Materials for Industrial End Uses.

\section{REFERENCES}

1. J. Aburto, S. Thiebaud, I. Alric, E. Borredon, D. Bikiaris, J. Prinos, and C. Panayiotou, Carbohydr. Polym., 34, 101 (1998).

2. D. Montane, X. Farriol, J. Salvado, P. Jollez, and E. Chornet, Biomass and Bioenery, 14, 261 (1998).

3. B. M. Gatewood, J. C. Wu, A. S. Roberts, A. C. Lumley, and A. M. Lewis, Textile Chemist and Colorist, 30, 39 (1998).

4. J. M. Lawther, R. C. Sun, and W. B. Banks, J. Agric. Food Chem., 43, 667 (1995).

5. R. Kohn, Z. Hromadkova, and A. Ebringerova, Collection Czechoslovak Chem. Commun., 51, 2250 (1986).

6. C. P. J. Glaudemans and T. E. Timell, Svensk Papperstidn., 61, 1 (1958).

7. T. E. Timell, Adv. Carbohyd. Chem., 19, 247 (1964).

8. B. Philipp, W. Wagenknecht, and I. Nehls, in "Cellulose, Structural and Fundational Aspects," J. F. Kennedy, G. O. Philipps, and P. A. Williams, Ed., Ellis Horwood, Chichester, 1989, p 173.

9. N. A. El-Shinnawy and S. F. El-Kalyoubi, J. Appl. Polym. Sci.,
30, 2171 (1988)

10. C. L. McCormick and P. A. Callais, Polymer, 28, 2317 (1987).

11. M. Shigematsu, A. Goto, S. Yoshida, M. Tanahashi, and Y. Shinoda, Mokuzai Gakkaishi, 40, 1214 (1994).

12. J. F. Carson and W. D. Maclay, J. Am. Chem. Soc., 70, 293 (1948).

13. A. B. Blakeney, P. J. Harris, R. J. Henry, and B. A. Stone, Carbohydr. Res., 113, 291 (1983).

14. R. C. Sun., J. M. Fang, P. Rowlands, and J. Bolton, J. Agric. Food Chem., 46, 2804 (1998).

15. R. C. Sun, J. M. Lawther, and W. B. Banks, Ind. Crops Prod., 4, 127 (1995).

16. R. C. Sun, J. M. Lawther, and W. B. Banks, Carbohydr. Polym., 29, 325 (1996).

17. B. Focher, A. Marzetti, A. Naggi, and G. Torri, Makromol. Chem., 190, 129 (1989).

18. D. L. Williams, H. A. Pretus, R. B. McNamee, E. L. Jones, H E. Ensley, and I. W. Browder, Carbohydr. Res., 235, 247 (1992).

19. A. Kato, J. Azuma, and T. Koshijima, Agric. Biol. Chem., 51, 1691 (1987)

20. A. Ebringerova, Z. Hromadkova, J. Alfoldi, and G. Berth, Carbohydr. Polym., 19, 99 (1992).

21. T. Imamura, T. Watanabe, M. Kuwahara, and T. Koshijima, Phytochemistry, 37, 1165 (1994).

22. M. I. Khalil, A. Hashem, and A. Hebeish, Starch/Stärke, 47, 394 (1995).

23. S. Gupta, R. N. Madan, and M. C. Bansal, Tappi J., 70, 113 (1987)

24. C. Q. Yang and X. Wang, Textile Res. J., 66, 595 (1996).

25. K. Rahn, M. Diamantoglou, D. Klemm, H. Berghmans, and T. Heinze, Die Angew. Makromol. Chem., 238, 143 (1996).

26. S. Lepeniotis and B. I. Feuer, Chemometrics and Intelligent Laboratory Systems, 36, 229 (1997). 